Kragujevac Journal of Mathematics

Volume 45(6) (2021), Pages 951-968.

\title{
A NEW METHOD TO SOLVE DUAL SYSTEMS OF FRACTIONAL INTEGRO-DIFFERENTIAL EQUATIONS BY LEGENDRE WAVELETS
}

\author{
RAZIEH KAVEHSARCHOGHA ${ }^{1}$, REZA EZZATI ${ }^{2 *}$, NASRIN KARAMIKABIR ${ }^{1}$, \\ AND FARAJOLLAH MOHAMMADI YAGHOBBI ${ }^{1}$
}

\begin{abstract}
The method that will be presented, is numerical solution based on the Legendre wavelets for solving dual systems of fractional integro-differential equations (FIDEs). First of all we make the operational matrix of fractional order integration. The application of this matrix is transforming FIDEs to a system of algebric equations. By this changing, we are able to solve it by a simple solution. In this way, the Legendre wavelets and their operator matrix are the most important keys of our solution. After explaining the method we test on some illustrative examples which numerical solutions of these examples demonstrate the validity and applicability of suggested method.
\end{abstract}

\section{INTRODUCTION}

Nowadays using fractional calculus has valuable usages in some fields of science and engineering. The study of dual systems of FIDEs have many applications in engineering, biomechanice and other scientific divisions. Dual systems of FIDEs also appear in modeling some of chemical and material engineering processes $[8,13,15]$. In most cases obtaining an analytical solution of FIDEs is impossible or so difficult. Thus, various procedures for obtaining approximate solutions of this kind of equations have attracted the attentions of many researchers.

In recent years, several numerical methods have been devoted for solving FIDEs but they are not properly applied to solve dual systems of FIDEs $[1,4,18]$. The greatest information that we can obtain from this case, is studing of papers that have

Key words and phrases. Legendre wavelets, fractional integro-differential equations, algebraic, dual systems.

2010 Mathematics Subject Classification. Primary: 26A33. Secondary: 65T60.

DOI 10.46793/KgJMat2106.951K

Received: April 04, 2019.

Accepted: July 08, 2019. 
been presented by various methods to arrive to an approximate solution. One of these methods is wavelet method [20]. Wavelets are generally a family of oscillatory functions which can be used to obtain approximate solutions of unknown functions [12]. There are many methods for solving FIDEs, by helping of wavelets; for instance take a look at $[2,9,11,22]$. The application of wavelets is significant in many scientific disciplines, such as time-frequency analysis, signal processing and numerical analysis [3].

This paper is based on Legendre wavelets that are a special type of wavelets that successfully have passed the exams in system analysis, system identification, optimal control and numerical solutions of differential and integral equations. Legendre wavelets are based on Legendre polynomials. From numerical point of view, wavelets have a closer and more accurate approximation than Legendre polynomials [17]. In the study of various methods for numerical solution of systems of FIDEs, we find that the wavelets method has been used less. Therefore, we have chosen the method of the Legendre wavelets for numerical solution of systems of FIDEs. We now apply the Legendre wavelets method to solve the following dual system [21]:

$$
\left\{\begin{array}{l}
D^{r} f(x)=u_{1}(x, f(x), g(x))+\int_{0}^{x} u_{2}(t, f(t), g(t)) d t, \\
D^{s} g(x)=v_{1}(x, f(x), g(x))+\int_{0}^{x} v_{2}(t, f(t), g(t)) d t,
\end{array}\right.
$$

where $x, t \in[0,1], r, s \in(0,1]$, and $D^{r}, D^{s}$ display the Caputo derivative operator.

\section{Legendre Wavelets and their Functional Properties}

2.1. Legendre wavelets. Legendre wavelets are defined on $[0,1)$ as [10]:

$$
\psi_{n m}(x)= \begin{cases}2^{\frac{k}{2}} \sqrt{m+\frac{1}{2}} L_{m}\left(2^{k} x-\widehat{n}\right), & \frac{\widehat{n}-1}{2^{k}} \leq x<\frac{\widehat{n}+1}{2^{k}}, \\ 0, & \text { otherwise }\end{cases}
$$

where $n=1,2, \ldots, 2^{k-1}, \widehat{n}=2 n-1, m=0,1,2, \ldots, M-1, k, M \in \mathbb{N}, m$ is the degree of the Legendre polynomials and $L_{m}(x)$ are the well-known Legendre polynomials of order $m$ that are defined on the interval $[-1,1]$ and satisfy the following recursive formula

$$
\begin{aligned}
& L_{0}(x)=1, \quad L_{1}(x)=x \\
& L_{m+1}(x)=\left(\frac{2 m+1}{m+1}\right) x L_{m}(x)-\left(\frac{m}{m+1}\right) L_{m-1}(x), \quad m=1,2, \ldots
\end{aligned}
$$

2.2. Function approximation. The Legendre wavelet series representation of the function $f(x)$ defined over $[0,1)$ is given by

$$
f(x)=\sum_{n=1}^{\infty} \sum_{m=0}^{\infty} a_{n m} \psi_{n m}(x)=A^{T} \Psi(x)
$$


where $a_{n m}=\left\langle f(x), \psi_{n m}(x)\right\rangle$ and $\langle\cdot, \cdot\rangle$ denotes the inner product. If the infinite series in (2.1) is finited, (2.1) can be written as

$$
f(x) \approx \sum_{n=1}^{2^{k-1}} \sum_{m=0}^{M-1} a_{n m} \psi_{n m}(x)=A^{T} \Psi(x),
$$

where $A$ and $\Psi(x)$ are $2^{k-1} M \times 1$ matrices given by

$$
\begin{aligned}
A= & {\left[a_{10}, a_{11}, \ldots, a_{1(M-1)}, a_{20}, a_{21}, \ldots, a_{2(M-1)}, \ldots, a_{2^{k-1} 0}, a_{2^{k-1} 1}, \ldots, a_{2^{k-1}(M-1)}\right]^{T} } \\
\Psi(x)= & {\left[\psi_{10}(x), \psi_{11}(x), \ldots, \psi_{1(M-1)}(x), \psi_{20}(x), \psi_{21}(x), \ldots, \psi_{2(M-1)}(x), \ldots,\right.} \\
& \left.\psi_{2^{k-1} 0}(x), \psi_{2^{k-1} 1}(x), \ldots, \psi_{2^{k-1}(M-1)}(x)\right]^{T} .
\end{aligned}
$$

For simplicity, (2.2) can be rewritten as

$$
f(x) \approx \sum_{i=1}^{n^{\prime}} a_{i} \psi_{i}(x)=A_{n^{\prime}}^{T} \Psi_{n^{\prime}}(x)=\widehat{f}(x),
$$

where $a_{i}=a_{n m}, \psi_{i}=\psi_{n m}, n^{\prime}=2^{k-1} M, i=M(n-1)+m+1$. Obtain the collocation points as

$$
x_{i}=\frac{i-0.5}{n^{\prime}}, \quad i=1,2, \cdots, 2^{k-1} M .
$$

We define the Legendre wavelets matrix as

$$
\phi_{n^{\prime} \times n^{\prime}}=\left[\Psi\left(\frac{1}{2 n^{\prime}}\right), \Psi\left(\frac{3}{2 n^{\prime}}\right), \Psi\left(\frac{5}{2 n^{\prime}}\right), \ldots, \Psi\left(\frac{i-0.5}{n^{\prime}}\right)\right] .
$$

\section{Operational Matrix of the Integration for Legendre Wavelets}

3.1. Preliminaries and natations. In this section, we first present some definitions and basic concepts that have the most applications in this paper [19].

Definition 3.1. The Reimann-Liouville fractional integral operator of order $\gamma \geq 0$ is a function defined as

$$
I^{\gamma} f(x)= \begin{cases}\frac{1}{\Gamma(\gamma)} \int_{0}^{x}(x-t)^{\gamma-1} f(t) d t, & \gamma>0 \\ f(x), & \gamma=0\end{cases}
$$

where $\Gamma(\gamma)$ is the gamma function as

$$
\Gamma(\gamma)=\int_{0}^{\infty} t^{\gamma-1} e^{-t} d t
$$

Definition 3.2. The Caputo fractional derivative of order $\gamma>0$ is defined as

$$
D^{\gamma} f(x)= \begin{cases}\frac{1}{\Gamma(n-\gamma)} \int_{0}^{x}(x-t)^{n-\gamma-1} f^{(n)}(t) d t, & \gamma>0, n-1<\gamma<n, \\ \frac{d^{(n)} f(x)}{d x^{n}}, & \gamma=n,\end{cases}
$$


where $x \in[0, \infty)$, and $n=1,2,3, \ldots$

For $x>0$ the Caputo derivative and Reimann-Liouville integral operator have the following relationships

$$
\begin{aligned}
& D^{\gamma} I^{\gamma} f(x)=f(x), \\
& I^{\gamma} D^{\gamma} f(x)=f(x)-\sum_{m=0}^{n-1} \frac{f^{(m)}\left(0^{+}\right)}{m !} x^{m}, \quad n-1<\gamma<n .
\end{aligned}
$$

3.2. Operational matrix of the fractional integration. Here the main goal is to get the fractional-order Legendre wavelets operational matrix of integration. For this purpose, we have to define the set of Block puls functions (BPFs) as follows [16]

$$
b_{i}(x)= \begin{cases}1, & \frac{i-1}{n^{\prime}} \leq x<\frac{i}{n^{\prime}} \\ 0, & \text { otherwise }\end{cases}
$$

where $i=1,2, \ldots, n^{\prime}$, and $n^{\prime}=2^{k-1} M$.

The BPFs have two properties which will be used later

$$
\begin{aligned}
b_{i}(x) b_{j}(x) & = \begin{cases}b_{i}(x), & i=j, \\
0, & i \neq j,\end{cases} \\
\int_{0}^{x} b_{i}(x) b_{j}(x) d x & = \begin{cases}\frac{1}{n^{\prime}}, & i=j, \\
0, & i \neq j .\end{cases}
\end{aligned}
$$

Definition 3.3. Let $C=\left[c_{1}, c_{2}, \ldots, c_{n^{\prime}}\right]^{T}$ and $D=\left[d_{1}, d_{2}, \ldots, d_{n^{\prime}}\right]^{T}$ be two matrices $n^{\prime} \times n^{\prime}$, then we define that $C \otimes D=\left[c_{1} d_{1}, c_{2} d_{2}, \ldots, c_{n^{\prime}} d_{n^{\prime}}\right]^{T}$.

Lemma 3.1. Suppose that $g(x)$ and $h(x)$ are two functions defined on $L^{2}[0,1]$ as we have $g(x)=G^{T} B_{n^{\prime}}(x)$ and $h(x)=H^{T} B_{n^{\prime}}(x)$, where $G^{T}=\left[g_{1}, g_{2}, \ldots, g_{n^{\prime}}\right], H^{T}=$ $\left[h_{1}, h_{2}, \ldots, h_{n^{\prime}}\right]$ and $B_{n^{\prime}}(x)=\left[b_{1}, b_{2}, \ldots, b_{n^{\prime}}\right]^{T}$, then we have

$$
\begin{aligned}
g(x) h(x) & \approx G^{T} B_{n^{\prime}}(x) H^{T} B_{n^{\prime}}(x)=\left(G^{T} \otimes H^{T}\right) B_{n^{\prime}}(x), \\
g(x)^{2} & \approx\left(G^{T} B_{n^{\prime}}(x)\right)^{2}=\left(G^{T}\right)^{2} B_{n^{\prime}}(x) .
\end{aligned}
$$

Proof. By using the properties of BPFs, the proof is obvious.

The fractional integration of order $\gamma$ in Reimann-Liouvill concept can be expressed as $[5]$

$$
I^{\gamma} B_{n^{\prime}}(x) \approx R^{\gamma} B_{n^{\prime}}(x)
$$


where $R^{\gamma}$ is the BPFs operational matrix with

$$
R^{\gamma}=\frac{1}{n^{\prime \gamma}} \frac{1}{\Gamma(\gamma+2)}\left[\begin{array}{ccccc}
1 & \xi_{1} & \xi_{2} & \cdots & \xi_{n^{\prime}-1} \\
0 & 1 & \xi_{1} & \cdots & \xi_{n^{\prime}-2} \\
0 & 0 & 1 & \cdots & \xi_{n^{\prime}-3} \\
0 & 0 & 0 & \cdots & \xi_{n^{\prime}-4} \\
\vdots & \vdots & \vdots & \ddots & \vdots \\
0 & 0 & 0 & \cdots & 1
\end{array}\right]
$$

and $\xi_{k}=(k+1)^{\gamma+1}-2 k^{\gamma+1}+(k-1)^{\gamma+1}, k=1,2, \ldots, n^{\prime}-1$.

We now derive the Legendre wavelets operational matrix of the fractional integration. The integration of Legendre wavelets $\Psi_{n^{\prime}}(x)$ can be obtained as

$$
I \Psi_{n^{\prime}}(x)=\int_{0}^{x} \Psi_{n^{\prime}}(\tau) d \tau \approx q_{n^{\prime} \times n^{\prime}} \Psi_{n^{\prime}}(x),
$$

where the $n^{\prime}$-square matrix $q_{n^{\prime} \times n^{\prime}}$ is called Legendre wavelets operational matrix and $q_{n^{\prime} \times n^{\prime}}^{\gamma}$ is called Legendre wavelets fractional integral operational matrix and achived by

$$
I^{\gamma} \Psi_{n^{\prime}}(x) \approx q_{n^{\prime} \times n^{\prime}}^{\gamma} \Psi(x)_{n^{\prime}},
$$

the Legendre wavelets can be expanded into $n^{\prime}$-set BPFs as

$$
\Psi_{n^{\prime}}(x) \approx \phi_{n^{\prime} \times n^{\prime}} B_{n^{\prime}}(x),
$$

we get [6] from (3.4), (3.6) and (3.7)

$$
\begin{aligned}
q_{n^{\prime} \times n^{\prime}}^{\gamma} \Psi_{n^{\prime}}(x) & \approx I^{\gamma} \Psi_{n^{\prime}}(x) \approx I^{\gamma} \phi_{n^{\prime} \times n^{\prime}} B_{n^{\prime}}(x)=\phi_{n^{\prime} \times n^{\prime}} I^{\gamma} B_{n^{\prime}}(x) \approx \phi_{n^{\prime} \times n^{\prime}} R^{\gamma} B_{n^{\prime}}(x) \\
& \approx \phi_{n^{\prime} \times n^{\prime}} R^{\gamma} \phi_{n^{\prime} \times n^{\prime}}^{-1} \psi_{n^{\prime}}(x) .
\end{aligned}
$$

Finally, we conclude from (3.6) $q_{n^{\prime} \times n^{\prime}}^{\gamma} \approx \phi_{n^{\prime} \times n^{\prime}} R^{\gamma} \Psi_{n^{\prime} \times n^{\prime}}^{-1}$.

In general, the matrix $\phi_{n^{\prime} \times n^{\prime}}$ counted in the below form

$$
\phi_{n^{\prime} \times n^{\prime}}=\left[\begin{array}{ccccc}
L & 0 & 0 & \cdots & 0 \\
0 & L & 0 & \cdots & 0 \\
0 & 0 & L & \cdots & 0 \\
\vdots & \vdots & \vdots & \ddots & \vdots \\
0 & 0 & 0 & \cdots & L
\end{array}\right]
$$

where $L$ is a $M \times M$ matrix given by [7]

$$
L=\left[\begin{array}{cccc}
\psi_{10}\left(\frac{1}{2 n^{\prime}}\right) & \psi_{10}\left(\frac{3}{2 n^{\prime}}\right) & \cdots & \psi_{10}\left(\frac{i-0.5}{n^{\prime}}\right) \\
\psi_{11}\left(\frac{1}{2 n^{\prime}}\right) & \psi_{11}\left(\frac{3}{2 n^{\prime}}\right) & \cdots & \psi_{11}\left(\frac{i-0.5}{n^{\prime}}\right) \\
\vdots & \vdots & \ddots & \vdots \\
\psi_{2^{k-1}(M-1)}\left(\frac{1}{2 n^{\prime}}\right) & \psi_{2^{k-1}(M-1)}\left(\frac{3}{2 n^{\prime}}\right) & \cdots & \psi_{2^{k-1}(M-1)}\left(\frac{i-0.5}{n^{\prime}}\right)
\end{array}\right]
$$


The six basis functions are by

$$
\begin{aligned}
& \left\{\begin{array}{l}
\psi_{10}(x)=\sqrt{2} \\
\psi_{11}(x)=\sqrt{6}(4 x-1), \\
\psi_{12}(x)=\sqrt{10}\left(24 x^{2}-12 x+1\right),
\end{array}\right. \\
& \left\{\begin{array}{l}
\psi_{20}(x)=\sqrt{2} \\
\psi_{21}(x)=\sqrt{6}(4 x-1), \\
\psi_{22}(x)=\sqrt{10}\left(24 x^{2}-36 x+13\right) .
\end{array}\right.
\end{aligned}
$$

Here, we present the matrices $R^{\gamma}, \phi_{n^{\prime} \times n^{\prime}}$ and $q^{\gamma}$ for $k=2, M=3, n=1,2$, $m=0,1,2, \gamma=0.6$ and using the collocation points $x_{i}=\frac{i-0.5}{n^{\prime}}, i=1,2, \ldots, n^{\prime}$, $n^{\prime}=2^{k-1} M$. Clearly, we have:

$$
\begin{aligned}
R^{0.6}= & {\left[\begin{array}{ccccccc}
0.23872 & 0.24622 & 0.17586 & 0.14847 & 0.13201 & 0.12061 \\
0 & 0.23872 & 0.24622 & 0.17586 & 0.14847 & 0.13201 \\
0 & 0 & 0.23872 & 0.24622 & 0.17586 & 0.14847 \\
0 & 0 & 0 & 0.23872 & 0.24622 & 0.17586 \\
0 & 0 & 0 & 0 & 0.23872 & 0.24622 \\
0 & 0 & 0 & 0 & 0 & 0.23872
\end{array}\right], } \\
\phi_{6 \times 6}= & {\left[\begin{array}{ccccccc}
1.41421 & 1.41421 & 1.41421 & 0 & 0 & 0 \\
-1.63299 & 0 & 1.63299 & 0 & 0 & 0 \\
0.52705 & -1.58114 & 0.52705 & 0 & 0 & 0 \\
0 & 0 & 0 & 1.41421 & 1.41421 & 1.41421 \\
0 & 0 & 0 & -1.63299 & 0 & 1.63299 \\
0 & 0 & 0 & 0.52705 & -1.58114 & 0.52705
\end{array}\right], } \\
q^{0.6}= & {\left[\begin{array}{ccccccc}
0.45856 & 0.18277 & -0.02360 & 0.47845 & -0.07337 & 0.01977 \\
-0.14723 & 0.15079 & 0.12261 & 0.06705 & -0.03495 & 0.01469 \\
-0.05571 & -0.09082 & 0.10681 & -0.04913 & 0.00096 & 0.00190 \\
0 & 0 & 0 & 0.45856 & 0.18277 & -0.02360 \\
0 & 0 & 0 & -0.14723 & 0.15079 & 0.12261 \\
0 & 0 & 0 & -0.05571 & -0.09082 & 0.10681
\end{array}\right] . }
\end{aligned}
$$

3.3. Error analysis. The following theorem presents the error analysis of the Legendre wavelets approximation function. By increasing values of $k$ and $M$ the error gets closer to zero. As you will see, solved examples confirm this sentence. So, we say surely the mentioned method and its approximation function will be successfully responsive for solving examples of the discussed subject.

Theorem 3.1 ([14]). Suppose $f(x) \in C^{2}[0,1]$ and $\widehat{f}(x)$ is the best approximation of $f(x)$, then we have for these two functions defined in (2.1) and (2.2):

$$
\begin{aligned}
\left\|e_{f}\right\|_{2} & =\|\operatorname{error}(f(x))\|_{2}=\|f(x)-\widehat{f}(x)\|_{2}=o\left(\frac{1}{M ! 2^{M k}}\right), \\
& =\frac{c}{M ! 2^{M k}} \text { c>0 } \text { as } k \rightarrow \infty, M \rightarrow \infty .
\end{aligned}
$$




\section{Numerical Examples}

In this section, we are going to solve two numerical examples by using the proposed method in Section 3, Also, we will compare their approximate and exact solution from graphical and numerical point of view. The numerical results show these performance of the mentioned method.

Example 4.1 ([21]).

$$
\begin{cases}D^{r} f(x)=-\frac{1}{2} f^{2}(x)-g(x)+\frac{1}{2}-\int_{0}^{x} g(t) f(t) d t, & 0<r \leq 1, \\ D^{s} g(x)=g^{2}(x)+f^{2}(x)-\int_{0}^{x} g(t) d t, & 0<s \leq 1,\end{cases}
$$

with the initial conditions $f(0)=1$ and $g(0)=0$. Exact solutions for the above coupled systems when $r=s=1$ are obtained by $f(x)=\cos x$ and $g(x)=\sin x$, the exact solutions of $f(x)$ and $g(x)$ for $r, s \in(0,1)$ are unknown.

Let

$$
\left\{\begin{array}{l}
D^{r} f(x) \approx A_{n^{\prime}}^{T} \Psi_{n^{\prime}}(x), \\
D^{s} g(x) \approx E_{n^{\prime}}^{T} \Psi_{n^{\prime}}(x),
\end{array}\right.
$$

where $A_{n^{\prime}}^{T}=\left[a_{1}, a_{2}, a_{3}, \ldots, a_{n^{\prime}}\right]$ and $E_{n^{\prime}}^{T}=\left[e_{1}, e_{2}, e_{3}, \ldots, e_{n^{\prime}}\right]$. By using the initial conditions and (3.1), (3.6), (3.7) and (4.2), we have

$$
\left\{\begin{aligned}
f(x) & =I^{r} D^{r} f(x)+f(0) \approx A_{n^{\prime}}^{T} q_{n^{\prime} \times n^{\prime}}^{r} \Psi_{n^{\prime}}(x)+1 \\
& \approx A_{n^{\prime}}^{T} q_{n^{\prime} \times n^{\prime}}^{r} \phi_{n^{\prime} \times n^{\prime}} B_{n^{\prime}}(x)+[1, \ldots, 1]_{n^{\prime} \times n^{\prime}} \\
g(x) & =I^{s} D^{s} g(x)+g(0) \approx E_{n^{\prime}}^{T} q_{n^{\prime} \times n^{\prime}}^{s} \Psi_{n^{\prime}}(x) \approx E_{n^{\prime}} q_{n^{\prime} \times n^{\prime}}^{s} \phi_{n^{\prime} \times n^{\prime}} B_{n^{\prime}}(x) .
\end{aligned}\right.
$$

Then, by using (3.2), (3.3), (3.5) and (4.3), we obtain

$$
\begin{aligned}
\int_{0}^{x} g(t) d t \approx & \int_{0}^{x} E_{n^{\prime}}^{T} q_{n^{\prime} \times n^{\prime}}^{s} \Psi_{n^{\prime}}(t) d t \approx E_{n^{\prime}}^{T} q_{n^{\prime} \times n^{\prime}}^{1+s} \phi_{n^{\prime} \times n^{\prime}} B_{n^{\prime}}(x), \\
g(x) f(x) \approx & \left(E_{n^{\prime}}^{T} q_{n^{\prime} \times n^{\prime}}^{s} \phi_{n^{\prime} \times n^{\prime}} B_{n^{\prime}}(x)\right)\left(A_{n^{\prime}}^{T} q_{n^{\prime} \times n^{\prime}}^{r} \phi_{n^{\prime} \times n^{\prime}} B_{n^{\prime}}(x)+1\right) \\
= & \left(E_{n^{\prime}}^{T} q_{n^{\prime} \times n^{\prime}}^{s} \phi_{n^{\prime} \times n^{\prime}} \otimes A_{n^{\prime}}^{T} q_{n^{\prime} \times n^{\prime}}^{r} \phi_{n^{\prime} \times n^{\prime}}\right) B_{n^{\prime}}(x)+E_{n^{\prime}}^{T} q_{n^{\prime} \times n^{\prime}}^{s} \phi_{n^{\prime} \times n^{\prime}} B_{n^{\prime}}(x), \\
\int_{0}^{x} g(t) f(t) d t \approx & \int_{0}^{x}\left(E_{n^{\prime}}^{T} q_{n^{\prime} \times n^{\prime}}^{s} \phi_{n^{\prime} \times n^{\prime}} \otimes A_{n^{\prime}}^{T} q_{n^{\prime} \times n^{\prime}}^{r} \phi_{n^{\prime} \times n^{\prime}}\right) B_{n^{\prime}}(t) d t \\
& +\int_{0}^{x} E_{n^{\prime}}^{T} q_{n^{\prime} \times n^{\prime}}^{s} \phi_{n^{\prime} \times n^{\prime}} B_{n^{\prime}}(t) d t \\
= & \left(E_{n^{\prime}}^{T} q_{n^{\prime} \times n^{\prime}}^{s} \phi_{n^{\prime} \times n^{\prime}} \otimes A_{n^{\prime}}^{T} q_{n^{\prime} \times n^{\prime}}^{r} \phi_{n^{\prime} \times n^{\prime}}\right) \int_{0}^{x} B_{n^{\prime}}(t) d t \\
& +E_{n^{\prime}}^{T} q_{n^{\prime} \times n^{\prime}}^{s} \phi_{n^{\prime} \times n^{\prime}} \int_{0}^{x} B_{n^{\prime}}(t) d t
\end{aligned}
$$




$$
\begin{aligned}
\approx & \left(E_{n^{\prime}}^{T} q_{n^{\prime} \times n^{\prime}}^{s} \phi_{n^{\prime} \times n^{\prime}} \otimes A_{n^{\prime}}^{T} q_{n^{\prime} \times n^{\prime}}^{r} \phi_{n^{\prime} \times n^{\prime}}\right) \int_{0}^{x} \phi_{n^{\prime} \times n^{\prime}}^{-1} \Psi_{n^{\prime}}(t) d t \\
& +\left(E_{n^{\prime}}^{T} q_{n^{\prime} \times n^{\prime}}^{s} \phi_{n^{\prime} \times n^{\prime}}\right) \int_{0}^{x} \phi_{n^{\prime} \times n^{\prime}}^{-1} \Psi_{n^{\prime}}(t) d t \\
\approx & \left(E_{n^{\prime}}^{T} q_{n^{\prime} \times n^{\prime}}^{s} \phi_{n^{\prime} \times n^{\prime}} \otimes A_{n^{\prime}}^{T} q_{n^{\prime} \times n^{\prime}}^{r} \phi_{n^{\prime} \times n^{\prime}}\right) \phi_{n^{\prime} \times n^{\prime}}^{-1} q_{n^{\prime} \times n^{\prime}} \phi_{n^{\prime} \times n^{\prime}} B_{n^{\prime}}(x) \\
& +E_{n^{\prime}}^{T} q_{n^{\prime} \times n^{\prime}}^{1+s} \phi_{n^{\prime} \times n^{\prime}} B_{n^{\prime}}(x) .
\end{aligned}
$$

By replacing (3.7), (4.2)-(4.4) and (4.5) into (4.1), and also according to the properties of BPFs, we conclude

$$
\left\{\begin{aligned}
A_{n^{\prime}}^{T} \phi_{n^{\prime} \times n^{\prime}}= & -\frac{1}{2}\left(A_{n^{\prime}}^{T} q_{n^{\prime} \times n^{\prime}}^{r} \phi_{n^{\prime} \times n^{\prime}}\right)^{2}-A_{n^{\prime}}^{T} q_{n^{\prime} \times n^{\prime}}^{r} \phi_{n^{\prime} \times n^{\prime}} \\
& -E_{n^{\prime}}^{T} q_{n^{\prime} \times n^{\prime}}^{s} \phi_{n^{\prime} \times n^{\prime}}-\left(E_{n^{\prime}}^{T} q_{n^{\prime} \times n^{\prime}}^{s} \phi_{n^{\prime} \times n^{\prime}} \otimes A_{n^{\prime}}^{T} q_{n^{\prime} \times n^{\prime}}^{r} \phi_{n^{\prime} \times n^{\prime}}\right. \\
& \left.+E_{n^{\prime}}^{T} q_{n^{\prime} \times n^{\prime}}^{s} \phi_{n^{\prime} \times n^{\prime}}\right) \phi_{n^{\prime} \times n^{\prime}}^{-1} q_{n^{\prime} \times n^{\prime}} \phi_{n^{\prime} \times n^{\prime}}, \\
E_{n^{\prime}}^{T} \phi_{n^{\prime} \times n^{\prime}}= & \left(E_{n^{\prime}}^{T} q_{n^{\prime} \times n^{\prime}}^{s} \phi_{n^{\prime} \times n^{\prime}}\right)^{2}+\left(A_{n^{\prime}}^{T} q_{n^{\prime} \times n^{\prime}}^{r} \phi_{n^{\prime} \times n^{\prime}}\right)^{2}+2 A_{n^{\prime}}^{T} q_{n^{\prime} \times n^{\prime}}^{r} \phi_{n^{\prime} \times n^{\prime}} \\
& -E_{n^{\prime}}^{T} q_{n^{\prime} \times n^{\prime}}^{1+s} \phi_{n^{\prime} \times n^{\prime}}+[1,1, \ldots, 1]_{1 \times n^{\prime}} .
\end{aligned}\right.
$$

(4.6) is now a system of nonlinear algebric equations which is a transformed type of (4.1). It has $2 n^{\prime}$ unknown coefficients, $A_{i}$ and $E_{i}$, which we can find them and the numerical solutions of $f(x)$ and $g(x)$ by solving this system by presented numerical method.

The approximate solutions obtained by using the proposed method and also absolute error value for different values $k, M, r, s$ and $x$ in the Tables $1-3$ have been shown. From Tables 1-3 and Figures 1-5 we can see that by increasing $k$ and $M$ the numerical solutions converge to the exact solutions, specially when $r, s \rightarrow 1$.

TABLE 1. Numerical results of the Example 4.1 for $k=2, M=6$, $n^{\prime}=2^{k-1} M=12, i=1,2,3, \ldots, n^{\prime}$, and different values $r$ and $s$.

\begin{tabular}{|l|l|l|l|l|l|}
\hline$x_{i}=\frac{i-0.5}{n^{\prime}}$ & $\begin{array}{l}r=0.7, s=0.7 \\
f(x), g(x)\end{array}$ & $\begin{array}{l}r=0.8, s=0.8 \\
f(x), g(x)\end{array}$ & $\begin{array}{l}r=0.9, s=0.9 \\
f(x), g(x)\end{array}$ & $\begin{array}{l}r=1, s=1 \\
f(x), g(x)\end{array}$ & $\begin{array}{l}\text { Ecact solution } \\
f(x), g(x)\end{array}$ \\
\hline \hline$x_{1}=0.04167$ & $0.98729,0.11185$ & $0.99339,0.08097$ & $0.99660,0.05817$ & $0.99827,0.41546$ & $0.99913,0.04166$ \\
\hline$x_{2}=0.12500$ & $0.95593,0.24847$ & $0.97390,0.19959$ & $0.98486,0.15830$ & $0.99136,0.12435$ & $0.99220,0.12467$ \\
\hline$x_{3}=0.20833$ & $0.91290,0.34846$ & $0.94327,0.29703$ & $0.96398,0.24904$ & $0.97758,0.20629$ & $0.97838,0.20683$ \\
\hline$x_{4}=0.29167$ & $0.86384,0.42851$ & $0.90475,0.38190$ & $0.93530,0.33342$ & $0.95704,0.28680$ & $0.95777,0.28755$ \\
\hline$x_{5}=0.37500$ & $0.81126,0.49298$ & $0.86008,0.45627$ & $0.89968,0.41195$ & $0.92988,0.36531$ & $0.93051,0.36627$ \\
\hline$x_{6}=0.45833$ & $0.75698,0.54381$ & $0.81063,0.52091$ & $0.85786,0.48458$ & $0.89628,0.44128$ & $0.89679,0.44245$ \\
\hline$x_{7}=0.54167$ & $0.70249,0.58196$ & $0.75760,0.57604$ & $0.81054,0.55109$ & $0.85648,0.51418$ & $0.85685,0.51556$ \\
\hline$x_{8}=0.62500$ & $0.64909,0.60799$ & $0.70209,0.62168$ & $0.75844,0.61113$ & $0.81076,0.58351$ & $0.81096,0.58510$ \\
\hline$x_{9}=0.70833$ & $0.59794,0.62222$ & $0.64516,0.65768$ & $0.70224,0.66435$ & $0.75944,0.64878$ & $0.75945,0.65057$ \\
\hline$x_{10}=0.79167$ & $0.55009,0.62485$ & $0.58783,0.68380$ & $0.64269,0.71035$ & $0.70287,0.70954$ & $0.70266,0.71153$ \\
\hline$x_{11}=0.87500$ & $0.50650,0.61613$ & $0.53112,0.69978$ & $0.58050,0.74871$ & $0.64145,0.76535$ & $0.64100,0.76754$ \\
\hline$x_{12}=0.95833$ & $0.46802,0.59639$ & $0.47601,0.70530$ & $0.51646,0.77900$ & $0.57560,0.81583$ & $0.57488,0.81823$ \\
\hline
\end{tabular}


TABLE 2. Numerical results of the Example 4.1 for $k=6, M=3$, $n^{\prime}=2^{k-1} M=96, i=1,2,3, \ldots, n^{\prime}$, and different values $r$ and $s$

\begin{tabular}{|l|l|l|l|l|l|}
\hline$x_{i}=\frac{i-0.5}{n^{\prime}}$ & $\begin{array}{l}r=0.85, s=0.85 \\
f(x), g(x)\end{array}$ & $\begin{array}{l}r=0.9, s=0.9 \\
f(x), g(x)\end{array}$ & $\begin{array}{l}r=0.95, s=0.95 \\
f(x), g(x)\end{array}$ & $\begin{array}{l}r=1, s=1 \\
f(x), g(x)\end{array}$ & $\begin{array}{l}\text { Ecact solution } \\
f(x), g(x)\end{array}$ \\
\hline \hline$x_{8}=0.07813$ & $0.99150,0.12072$ & $0.99393,0.10459$ & $0.99568,0.09043$ & $0.99694,0.07804$ & $0.99695,0.07805$ \\
\hline$x_{16}=0.16146$ & $0.97100,0.22197$ & $0.97769,0.19983$ & $0.98292,0.17943$ & $0.98698,0.16075$ & $0.98699,0.16076$ \\
\hline$x_{24}=0.24479$ & $0.94160,0.31225$ & $0.95307,0.28786$ & $0.96250,0.26449$ & $0.97018,0.24234$ & $0.97019,0.24235$ \\
\hline$x_{32}=0.32813$ & $0.90481,0.39397$ & $0.22105,0.36986$ & $0.93492,0.34583$ & $0.94664,0.32226$ & $0.94665,0.32227$ \\
\hline$x_{40}=0.41146$ & $0.86177,0.46779$ & $0.88241,0.44602$ & $0.90064,0.42322$ & $0.91653,0.39993$ & $0.91654,0.39995$ \\
\hline$x_{48}=0.49479$ & $0.81349,0.53383$ & $0.83787,0.51619$ & $0.86010,0.49628$ & $0.88006,0.47483$ & $0.88007,0.47485$ \\
\hline$x_{56}=0.57813$ & $0.76090,0.59198$ & $0.78813,0.58007$ & $0.81378,0.56461$ & $0.83748,0.54643$ & $0.83749,0.54645$ \\
\hline$x_{64}=0.66146$ & $0.70490,0.64202$ & $0.73389,0.63733$ & $0.76217,0.62776$ & $0.78910,0.61424$ & $0.78910,0.61427$ \\
\hline$x_{72}=0.74479$ & $0.64638,0.68365$ & $0.67585,0.68758$ & $0.70579,0.68530$ & $0.73523,0.67779$ & $0.73523,0.67782$ \\
\hline$x_{80}=0.82813$ & $0.58625,0.71652$ & $0.61477,0.73042$ & $0.64520,0.73680$ & $0.67626,0.73663$ & $0.67626,0.73666$ \\
\hline$x_{88}=0.91146$ & $0.52539,0.74023$ & $0.55138,0.76544$ & $0.58097,0.78185$ & $0.61260,0.79036$ & $0.61259,0.79040$ \\
\hline$x_{96}=0.99979$ & $0.46474,0.75438$ & $0.48647,0.79219$ & $0.51373,0.82002$ & $0.54469,0.83861$ & $0.54468,0.83865$ \\
\hline
\end{tabular}

TABLE 3. Absolute error relevant to Tables 1 and 2 when $r=s=1$

\begin{tabular}{|l|l|l||l|l|l|}
\hline \hline$x_{i}$ & $e_{f}$ & $e_{g}$ & $x_{i}$ & $e_{f}$ & $e_{g}$ \\
\hline \hline$x_{1}$ & $8.6311 e-04$ & $1.0824 e-04$ & $x_{8}$ & $1.3409 e-05$ & $3.1776 e-06$ \\
\hline$x_{2}$ & $8.4048 e-04$ & $3.2442 e-04$ & $x_{16}$ & $1.2897 e-05$ & $6.5587 e-06$ \\
\hline$x_{3}$ & $7.9451 e-04$ & $5.3968 e-04$ & $x_{24}$ & $1.2015 e-05$ & $9.9213 e-06$ \\
\hline$x_{4}$ & $7.2457 e-04$ & $7.5337 e-04$ & $x_{32}$ & $1.0753 e-05$ & $1.3255 e-05$ \\
\hline$x_{5}$ & $6.3022 e-04$ & $9.6491 e-04$ & $x_{40}$ & $9.1071 e-06$ & $1.6552 e-05$ \\
\hline$x_{6}$ & $5.1122 e-04$ & $1.1739 e-03$ & $x_{48}$ & $7.0736 e-06$ & $1.9807 e-05$ \\
\hline$x_{7}$ & $3.6749 e-04$ & $1.3801 e-03$ & $x_{56}$ & $4.6529 e-06$ & $2.3020 e-05$ \\
\hline$x_{8}$ & $1.9913 e-04$ & $1.5838 e-03$ & $x_{64}$ & $1.8473 e-06$ & $2.6198 e-05$ \\
\hline$x_{9}$ & $6.3200 e-06$ & $1.7858 e-03$ & $x_{72}$ & $1.3399 e-06$ & $2.9357 e-05$ \\
\hline$x_{10}$ & $2.1069 e-04$ & $1.9875 e-03$ & $x_{80}$ & $4.9047 e-06$ & $3.2527 e-05$ \\
\hline$x_{11}$ & $4.5169 e-04$ & $2.1913 e-03$ & $x_{88}$ & $8.8442 e-06$ & $3.5751 e-05$ \\
\hline$x_{12}$ & $7.1658 e-04$ & $2.4006 e-03$ & $x_{96}$ & $1.3158 e-05$ & $3.9088 e-05$ \\
\hline \hline
\end{tabular}

Example $4.2([21])$.

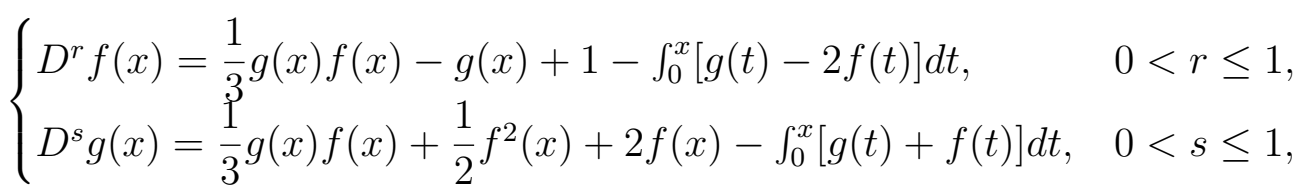

with the initial conditions $f(0)=0$ and $g(0)=0$, exact solutions for the above coupled systems when $r=s=1$ are obtained by $f(x)=x$ and $g(x)=x^{2}$. The exact solutions 

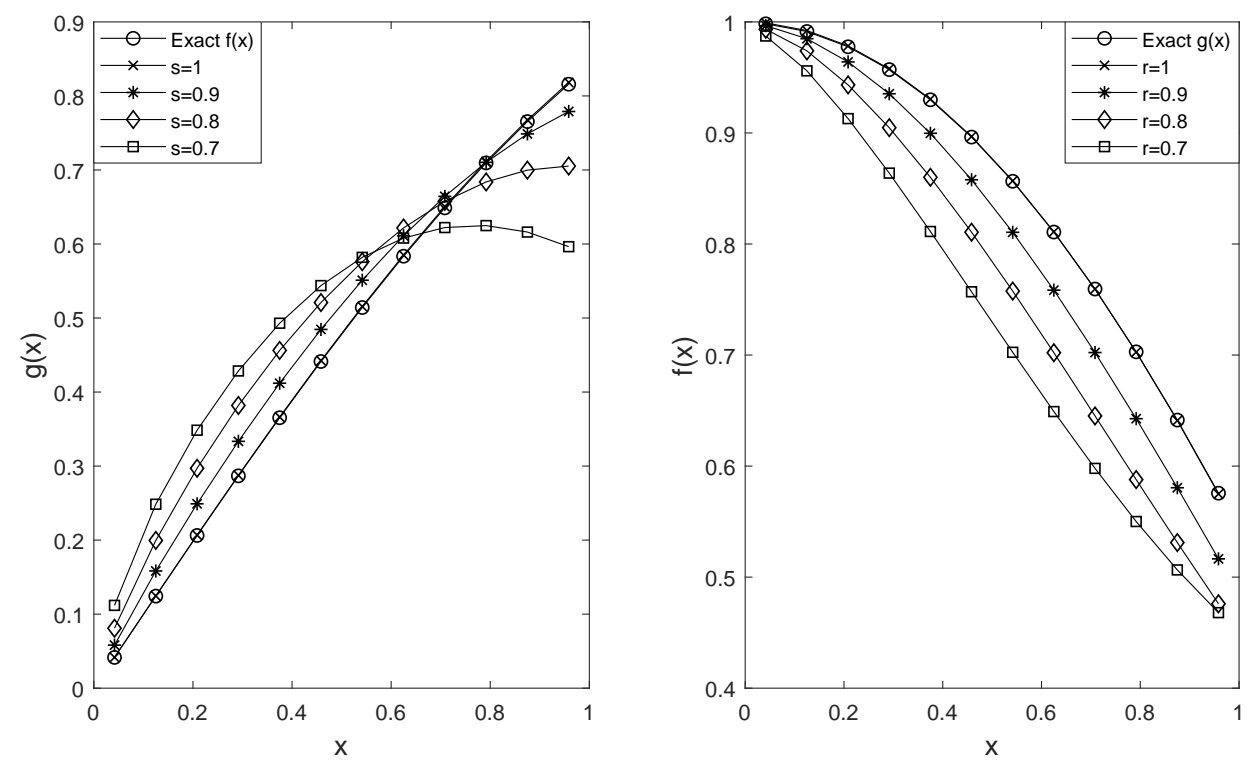

FiguRE 1. Numerical solution for different values of $r$ and $s$ when $k=2, M=6$ and $n^{\prime}=12$.
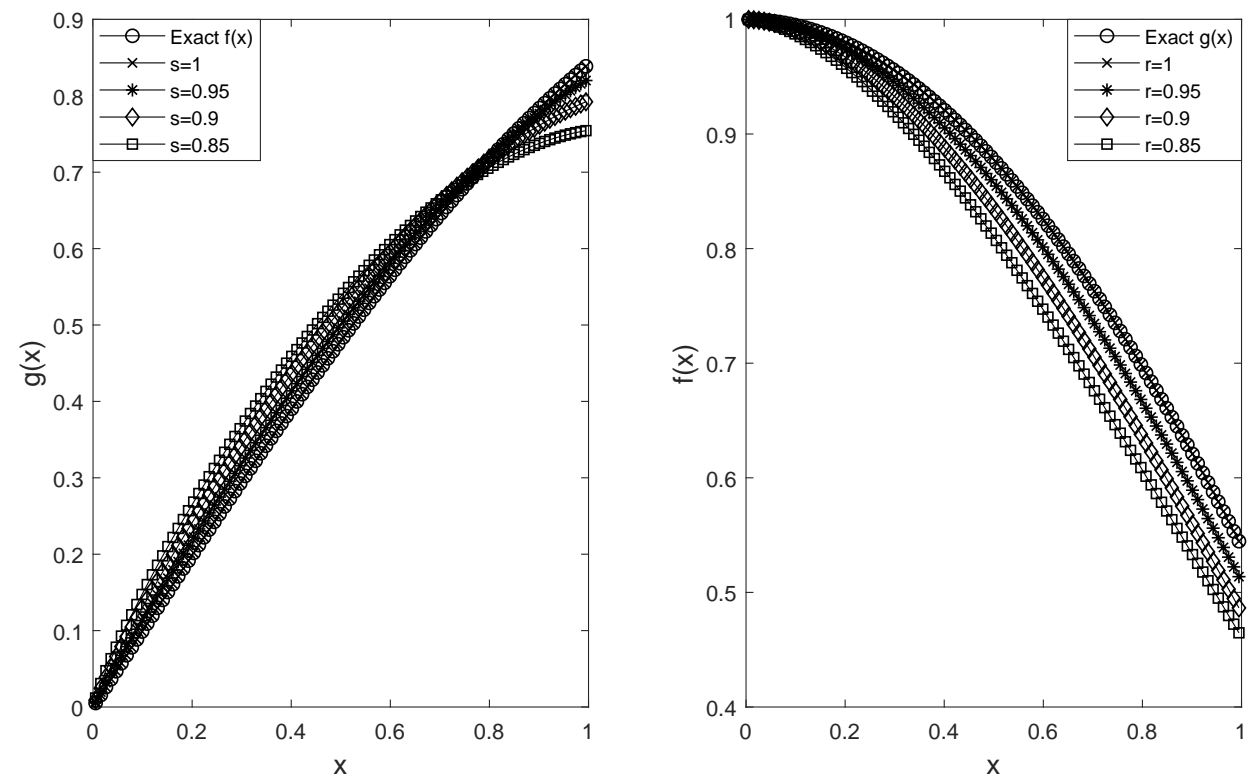

FiguRe 2. Numerical solution for different values of $r$ and $s$ when $k=6, M=3$ and $n^{\prime}=96$. 

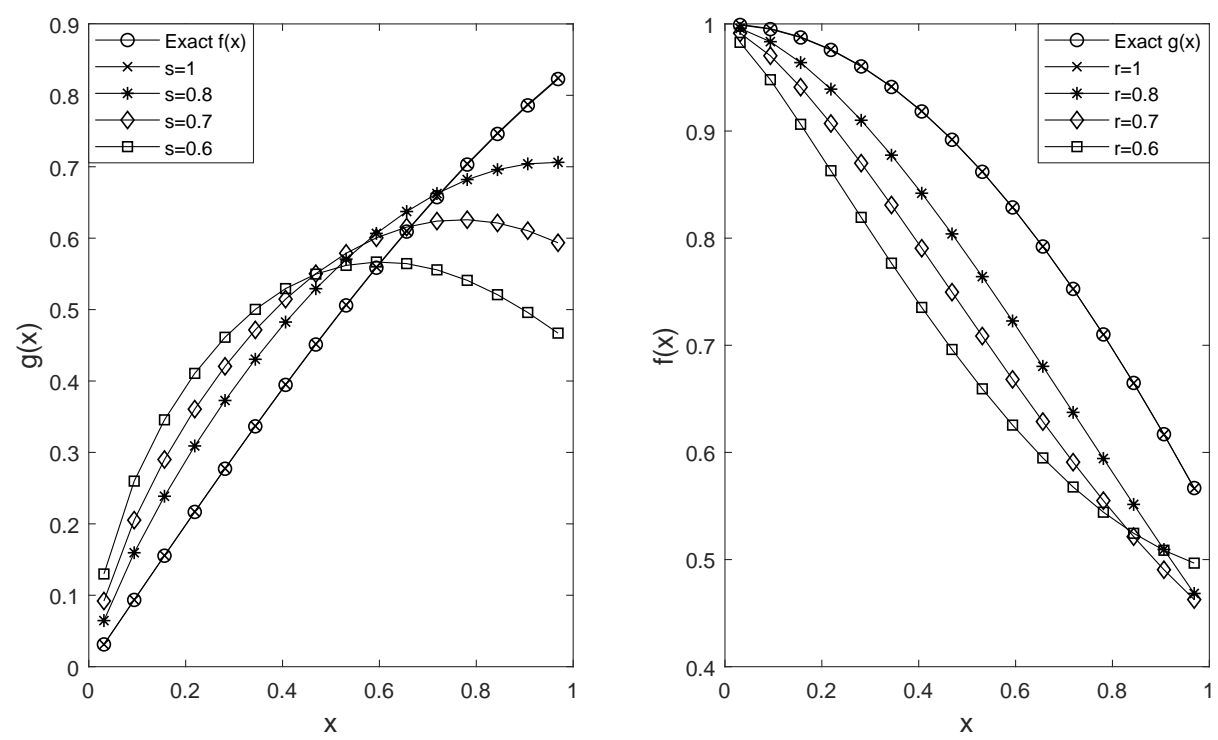

FiguRE 3. Numerical solution for different values of $r$ and $s$ when $k=3, M=4$ and $n^{\prime}=16$.
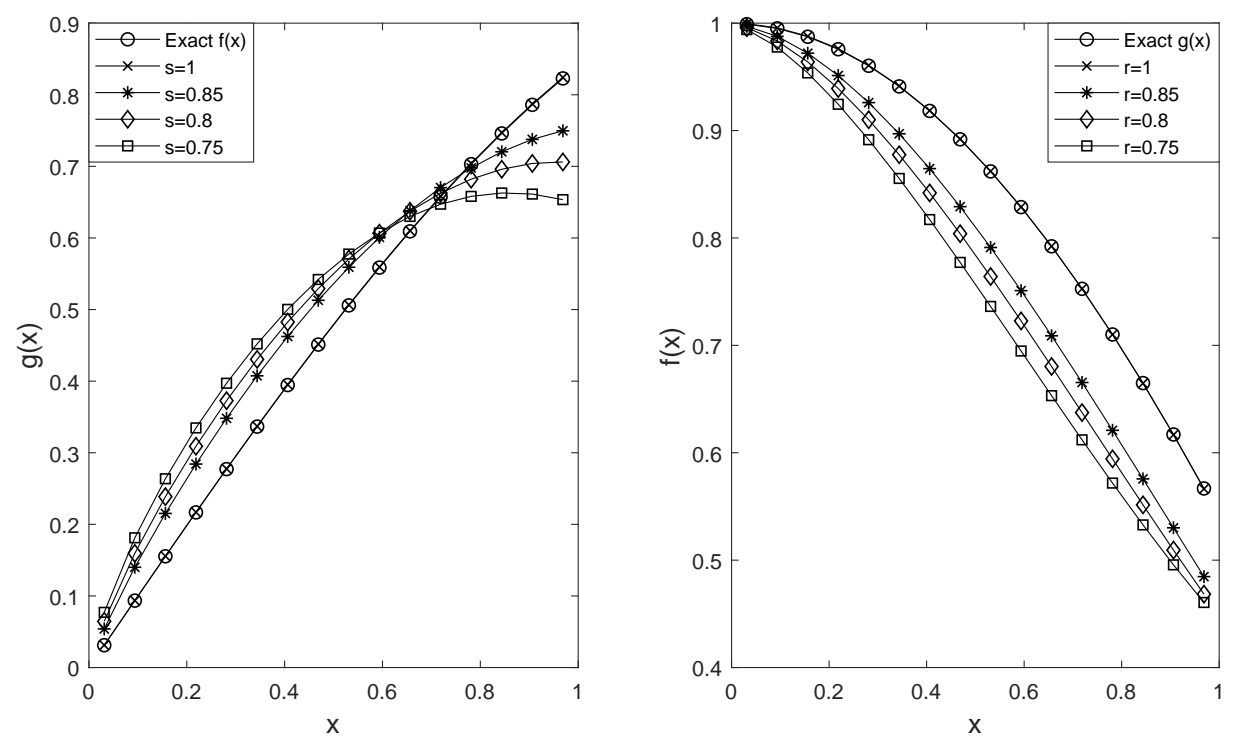

Figure 4. Numerical solution for different values of $r$ and $s$ when $k=3, M=4$ and $n^{\prime}=16$. 

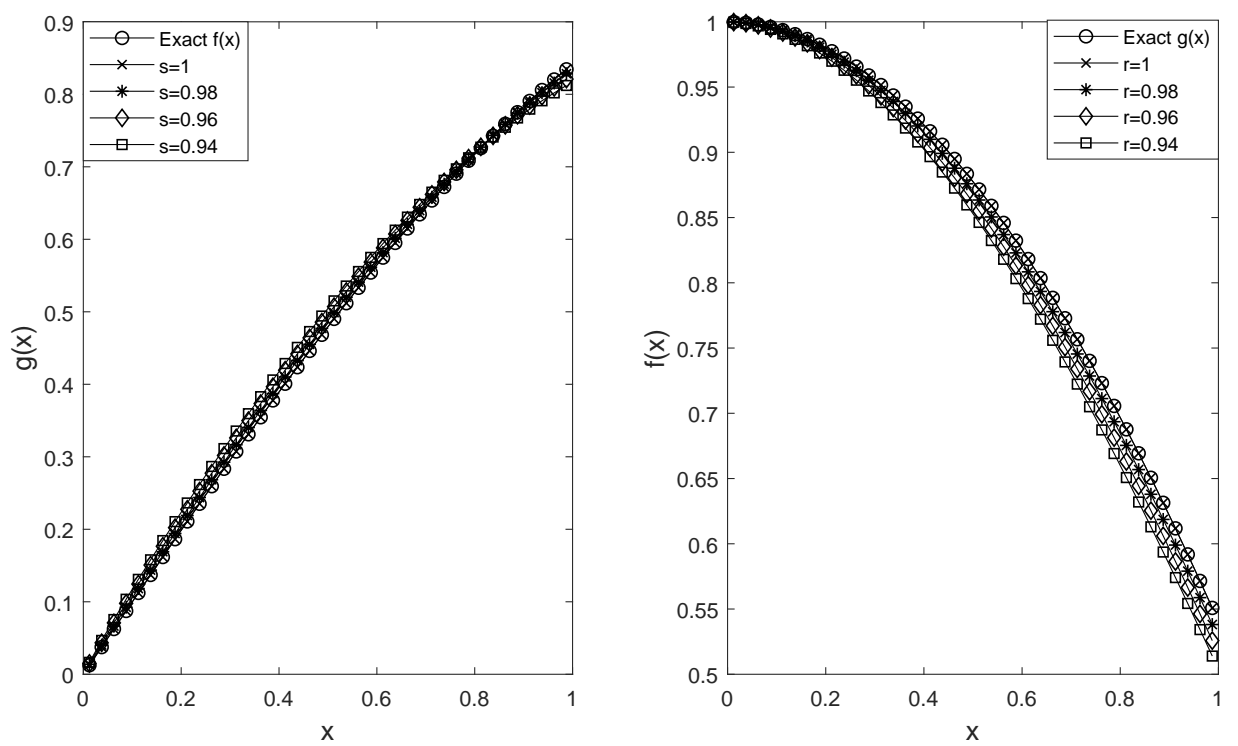

Figure 5. Numerical solution for different values of $r$ and $s$ when $k=4, M=5$ and $n^{\prime}=40$.

of $f(x)$ and $g(x)$ for $r, s \in(0,1)$ are unknown. Let

$$
\left\{\begin{array}{l}
D^{r} f(x) \approx A_{n^{\prime}}^{T} \Psi_{n^{\prime}}(x), \\
D^{s} g(x) \approx E_{n^{\prime}}^{T} \Psi_{n^{\prime}}(x),
\end{array}\right.
$$

where $A_{n^{\prime}}^{T}=\left[a_{1}, a_{2}, a_{3}, \ldots, a_{n^{\prime}}\right]$ and $E_{n^{\prime}}^{T}=\left[e_{1}, e_{2}, e_{3}, \ldots, e_{n^{\prime}}\right]$.

By using the initial conditions and (3.2), (3.6), (3.7) and (4.8) we have

$$
\left\{\begin{array}{l}
f(x)=I^{r} D^{r} f(x)+f(0) \approx A_{n^{\prime}}^{T} q_{n^{\prime} \times n^{\prime}}^{r} \Psi_{n^{\prime}}(x) \approx A_{n^{\prime}}^{T} q_{n^{\prime} \times n^{\prime}}^{r} \phi_{n^{\prime} \times n^{\prime}} B_{n^{\prime}}(x), \\
g(x)=I^{s} D^{s} g(x)+g(0) \approx E_{n^{\prime}}^{T} q_{n^{\prime} \times n^{\prime}}^{s} \Psi_{n^{\prime}}(x) \approx E_{n^{\prime}}^{T} q_{n^{\prime} \times n^{\prime}}^{s} \phi_{n^{\prime} \times n^{\prime}} B_{n^{\prime}}(x) .
\end{array}\right.
$$

So, by using (3.2), (3.3), (3.5) and (4.9), we obtain

$$
\begin{aligned}
g(x) f(x) & \approx\left(E_{n^{\prime}}^{T} q_{n^{\prime} \times n^{\prime}}^{s} \phi_{n^{\prime} \times n^{\prime}} B_{n^{\prime}}(x)\right)\left(A_{n^{\prime}}^{T} q_{n^{\prime} \times n^{\prime}}^{r} \phi_{n^{\prime} \times n^{\prime}} B_{n^{\prime}}(x)\right) \\
& =\left(E_{n^{\prime}}^{T} q_{n^{\prime} \times n^{\prime}}^{s} \phi_{n^{\prime} \times n^{\prime}} \otimes A_{n^{\prime}}^{T} q_{n^{\prime} \times n^{\prime}}^{r} \phi_{n^{\prime} \times n^{\prime}}\right) B_{n^{\prime}}(x), \\
f^{2}(x) & \approx\left(A_{n^{\prime}}^{T} q_{n^{\prime} \times n^{\prime}}^{r} \phi_{n^{\prime} \times n^{\prime}} B_{n^{\prime}}(x)\right)^{2}=\left(A_{n^{\prime}}^{T} q_{n^{\prime} \times n^{\prime}}^{r} \phi_{n^{\prime} \times n^{\prime}}\right)^{2} B_{n^{\prime}}(x), \\
\int_{0}^{x} f(t) d t & \approx \int_{0}^{x} A_{n^{\prime}}^{T} q_{n^{\prime} \times n^{\prime}}^{r} \Psi_{n^{\prime}}(t) d t=A_{n^{\prime}}^{T} q_{n^{\prime} \times n^{\prime}}^{r} \int_{0}^{x} \Psi_{n^{\prime}}(t) d t \\
& \approx A_{n^{\prime}}^{T} q_{n^{\prime} \times n^{\prime}}^{r} q_{n^{\prime} \times n^{\prime}}^{1} \Psi(x) \approx A_{n^{\prime}}^{T} q_{n^{\prime} \times n^{\prime}}^{1+r} \phi_{n^{\prime} \times n^{\prime}} B_{n^{\prime}}(x), \\
\int_{0}^{x} g(t) d t & \approx \int_{0}^{x} E_{n^{\prime}}^{T} q_{n^{\prime} \times n^{\prime}}^{s} \Psi_{n^{\prime}}(t) d t \approx E_{n^{\prime}}^{T} q_{n^{\prime} \times n^{\prime}}^{1+s} \phi_{n^{\prime} \times n^{\prime}} B_{n^{\prime}}(x) .
\end{aligned}
$$


By replacing (3.7) and (4.8)-(4.13) into (4.7), we obtain

$$
\left\{\begin{aligned}
A_{n^{\prime}}^{T} \phi_{n^{\prime} \times n^{\prime}} B_{n^{\prime}}(x)= & \frac{1}{3}\left(E_{n^{\prime}} q_{n^{\prime} \times n^{\prime}}^{s} \phi_{n^{\prime} \times n^{\prime}} \otimes A_{n^{\prime}}^{T} q_{n^{\prime} \times n^{\prime}}^{r} \phi_{n^{\prime} \times n^{\prime}}\right) B_{n^{\prime}}(x) \\
& -E_{n^{\prime}}^{T} q_{n^{\prime} \times n^{\prime}}^{s} \phi_{n^{\prime} \times n^{\prime}} B_{n^{\prime}}(x)+[1,1,1, \ldots, 1]_{1 \times n^{\prime}} B_{n^{\prime}}(x) \\
& -E_{n^{\prime}}^{T} q_{n^{\prime} \times n^{\prime}}^{1+s} \phi_{n^{\prime} \times n^{\prime}} B_{n^{\prime}}(x)+2 A_{n^{\prime}}^{T} q_{n^{\prime} \times n^{\prime}}^{1+r} \phi_{n^{\prime} \times n^{\prime}} B_{n^{\prime}}(x), \\
E_{n^{\prime}}^{T} \phi_{n^{\prime} \times n^{\prime}}=\quad & \frac{1}{3}\left(E_{n^{\prime}}^{T} q_{n^{\prime} \times n^{\prime}}^{s} \phi_{n^{\prime} \times n^{\prime}} \otimes A_{n^{\prime}}^{T} q_{n^{\prime} \times n^{\prime}}^{r} \phi_{n^{\prime} \times n^{\prime}}\right) B_{n^{\prime}}(x) \\
& +\frac{1}{2}\left(A_{n^{\prime}}^{T} q_{n^{\prime} \times n^{\prime}}^{r} \phi_{n^{\prime} \times n^{\prime}}\right)^{2} B_{n^{\prime}}(x)+2 A_{n^{\prime}}^{T} q_{n^{\prime} \times n^{\prime}}^{r} \phi_{n^{\prime} \times n^{\prime}} B_{n^{\prime}}(x) \\
& -E_{n^{\prime}}^{T} q_{n^{\prime} \times n^{\prime}}^{1+s} \phi_{n^{\prime} \times n^{\prime}} B_{n^{\prime}}(x)-A_{n^{\prime}}^{T} q_{n^{\prime} \times n^{\prime}}^{1+r} \phi_{n^{\prime} \times n^{\prime}} B_{n^{\prime}}(x) .
\end{aligned}\right.
$$

According to the properties of BPFs and (4.14) we have

$$
\left\{\begin{aligned}
A_{n^{\prime}}^{T} \phi_{n^{\prime} \times n^{\prime}}= & \frac{1}{3}\left(E_{n^{\prime}}^{T} q_{n^{\prime} \times n^{\prime}}^{s} \phi_{n^{\prime} \times n^{\prime}} \otimes A_{n^{\prime}}^{T} q_{n^{\prime} \times n^{\prime}}^{r} \phi_{n^{\prime} \times n^{\prime}}\right)-E_{n^{\prime}}^{T} q_{n^{\prime} \times n^{\prime}}^{s} \phi_{n^{\prime} \times n^{\prime}} \\
& +[1,1,1, \ldots, 1]_{1 \times n^{\prime}}-E_{n^{\prime}}^{T} q_{n^{\prime} \times n^{\prime}}^{1+s} \phi_{n^{\prime} \times n^{\prime}}+2 A_{n^{\prime}}^{T} q_{n^{\prime} \times n^{\prime}}^{1+r} \phi_{n^{\prime} \times n^{\prime}}, \\
E_{n^{\prime}}^{T} \phi_{n^{\prime} \times n^{\prime}}= & \frac{1}{3}\left(E_{n^{\prime}}^{T} q_{n^{\prime} \times n^{\prime}}^{s} \phi_{n^{\prime} \times n^{\prime}} \otimes A_{n^{\prime}}^{T} q_{n^{\prime} \times n^{\prime}}^{r} \phi_{n^{\prime} \times n^{\prime}}\right)+\frac{1}{2}\left(A_{n^{\prime}}^{T} q_{n^{\prime} \times n^{\prime}}^{r} \phi_{n^{\prime} \times n^{\prime}}\right)^{2} \\
& +2 A_{n^{\prime}}^{T} q_{n^{\prime} \times n^{\prime}}^{r} \phi_{n^{\prime} \times n^{\prime}}-E_{n^{\prime}}^{T} q_{n^{\prime} \times n^{\prime}}^{1+s} \phi_{n^{\prime} \times n^{\prime}}-A_{n^{\prime}}^{T} q_{n^{\prime} \times n^{\prime}}^{1+r} \phi_{n^{\prime} \times n^{\prime}} .
\end{aligned}\right.
$$

TABLE 4. Numerical results of the Example 4.2 for $k=2, M=6$, $n^{\prime}=12$ and different values $r$ and $s$.

\begin{tabular}{|l|l|l|l|l|l|}
\hline$x_{i}=\frac{i-0.5}{n^{\prime}}$ & $\begin{array}{l}r=0.7, s=0.7 \\
f(x), g(x)\end{array}$ & $\begin{array}{l}r=0.8, s=0.8 \\
f(x), g(x)\end{array}$ & $\begin{array}{l}r=0.9, s=0.9 \\
f(x), g(x)\end{array}$ & $\begin{array}{l}r=1, s=1 \\
f(x), g(x)\end{array}$ & $\begin{array}{l}\text { Ecact solution } \\
f(x), g(x)\end{array}$ \\
\hline \hline$x_{1}=0.04167$ & $0.11157,0.02570$ & $0.08101,0.01331$ & $0.05826,0.00681$ & $0.04162,0.00345$ & $0.04167,0.00174$ \\
\hline$x_{2}=0.12500$ & $0.24734,0.08992$ & $0.19985,0.05289$ & $0.15887,0.03048$ & $0.12485,0.01729$ & $0.12500,0.01563$ \\
\hline$x_{3}=0.20833$ & $0.34747,0.17865$ & $0.29849,0.11560$ & $0.25105,0.07284$ & $0.20808,0.04499$ & $0.20833,0.04340$ \\
\hline$x_{4}=0.29167$ & $0.43049,0.28039$ & $0.38662,0.19511$ & $0.33858,0.13148$ & $0.29130,0.08656$ & $0.29167,0.0851$ \\
\hline$x_{5}=0.37500$ & $0.50203,0.39010$ & $0.46725,0.28816$ & $0.42265,0.20501$ & $0.37452,0.14198$ & $0.37500,0.14063$ \\
\hline$x_{6}=0.45833$ & $0.56500,0.50429$ & $0.54192,0.39228$ & $0.50386,0.29232$ & $0.45773,0.21126$ & $0.45833,0.21007$ \\
\hline$x_{7}=0.54167$ & $0.62123,0.62019$ & $0.61160,0.50541$ & $0.58259,0.39247$ & $0.54094,0.29439$ & $0.54167,0.29340$ \\
\hline$x_{8}=0.62500$ & $0.67207,0.73553$ & $0.67698,0.62570$ & $0.65908,0.50455$ & $0.62415,0.39137$ & $0.62500,0.39063$ \\
\hline$x_{9}=0.70833$ & $0.71856,0.84840$ & $0.73855,0.75142$ & $0.73351,0.62770$ & $0.70736,0.50221$ & $0.70833,0.50174$ \\
\hline$x_{10}=0.79167$ & $0.76163,0.95723$ & $0.79675,0.88091$ & $0.80600,0.76104$ & $0.79057,0.62689$ & $0.79167,0.62674$ \\
\hline$x_{11}=0.87500$ & $0.80212,1.06071$ & $0.85194,1.01260$ & $0.87667,0.90368$ & $0.87377,0.76541$ & $0.87500,0.76563$ \\
\hline$x_{12}=0.95833$ & $0.84082,1.15789$ & $0.90445,1.14498$ & $0.94558,1.05471$ & $0.95697,0.91778$ & $0.95833,0.91840$ \\
\hline
\end{tabular}


TABLE 5. Numerical results of the Example 4.2 for $k=6, M=3$, $n^{\prime}=96$, for $i=8,16,24, \ldots, 96$, and different values $r$ and $s$.

\begin{tabular}{|l|l|l|l|l|l|}
\hline$x_{i}=\frac{i-0.5}{n^{\prime}}$ & $\begin{array}{l}r=0.85 \\
f(x), g(x)\end{array}$ & $\begin{array}{l}r=0.90 \\
f(x), g(x)\end{array}$ & $\begin{array}{l}r=0.95 \\
s=0.95 \\
f(x), g(x)\end{array}$ & $\begin{array}{l}s=1 \\
f(x), g(x)\end{array}$ & $\begin{array}{l}\text { Exact solution } \\
f(x), g(x)\end{array}$ \\
\hline \hline$x_{8}=0.07813$ & $0.12072,0.01712$ & $0.10466,0.01221$ & $0.09051,0.00867$ & $0.07812,0.00613$ & $0.07813,0.00610$ \\
\hline$x_{16}=0.16146$ & $0.22252,0.05872$ & $0.20055,, 0.45011$ & $0.18013,0.03434$ & $0.16146,0.02609$ & $0.16146,0.02607$ \\
\hline$x_{24}=0.24479$ & $0.31477,0.11879$ & $0.29056,0.09507$ & $0.26714,0.07568$ & $0.24479,0.05995$ & $0.24479,0.05992$ \\
\hline$x_{32}=0.32813$ & $0.40070,0.19461$ & $0.37657,0.16072$ & $0.35221,0.13191$ & $0.32812,0.10769$ & $0.32813,0.10767$ \\
\hline$x_{40}=0.41146$ & $0.48172,0.28420$ & $0.45943,0.24076$ & $0.43578,0.20252$ & $0.41145,0.16932$ & $0.41146,0.16930$ \\
\hline$x_{48}=0.49479$ & $0.55863,0.38597$ & $0.53961,0.33418$ & $0.51805,0.28702$ & $0.49478,0.24484$ & $0.49479,0.24482$ \\
\hline$x_{56}=0.57813$ & $0.63198,0.49845$ & $0.61741,0.44006$ & $0.59916,0.38499$ & $0.57811,0.33424$ & $0.57813,0.33423$ \\
\hline$x_{64}=0.66146$ & $0.70212,0.62030$ & $0.69306,0.55752$ & $0.67920,0.49602$ & $0.66144,0.43754$ & $0.66146,0.43753$ \\
\hline$x_{72}=0.74479$ & $0.76936,0.75018$ & $0.76670,0.68568$ & $0.78823,0.61968$ & $0.74478,0.55472$ & $0.74479,0.55471$ \\
\hline$x_{80}=0.82813$ & $0.83390,0.88680$ & $0.83845,0.82367$ & $0.83628,0.75556$ & $0.82811,0.68579$ & $0.82813,0.68579$ \\
\hline$x_{88}=0.91146$ & $0.89595,1.02884$ & $0.90839,0.97059$ & $0.91339,0.90321$ & $0.91144,0.83075$ & $0.91146,0.83076$ \\
\hline$x_{96}=0.99479$ & $0.95568,1.17499$ & $0.97659,1.12551$ & $0.98958,1.06215$ & $0.99477,0.98960$ & $0.99479,0.98961$ \\
\hline
\end{tabular}

TABLE 6. Absolute error relevant to Tables 4 and 5 when $r=s=1$

\begin{tabular}{|l|l|l||l|l|l|}
\hline \hline$x_{i}$ & $e_{f}$ & $e_{g}$ & $x_{i}$ & $e_{f}$ & $e_{g}$ \\
\hline \hline$x_{1}$ & $4.9582 e-05$ & $1.7179 e-03$ & $x_{8}$ & $1.4528 e-06$ & $2.6604 e-05$ \\
\hline$x_{2}$ & $1.5141 e-04$ & $1.6686 e-03$ & $x_{16}$ & $3.0835 e-06$ & $2.5657 e-05$ \\
\hline$x_{3}$ & $2.5823 e-04$ & $1.5927 e-03$ & $x_{24}$ & $4.7883 e-06$ & $2.4283 e-05$ \\
\hline$x_{4}$ & $3.6945 e-04$ & $1.4885 e-03$ & $x_{32}$ & $6.5581 e-06$ & $2.2451 e-05$ \\
\hline$x_{5}$ & $4.8451 e-04$ & $1.3539 e-03$ & $x_{40}$ & $8.3847 e-06$ & $2.0132 e-05$ \\
\hline$x_{6}$ & $6.0291 e-04$ & $1.1872 e-03$ & $x_{48}$ & $1.0261 e-05$ & $1.7294 e-05$ \\
\hline$x_{7}$ & $7.2421 e-04$ & $9.8598 e-04$ & $x_{56}$ & $1.2179 e-05$ & $1.3901 e-05$ \\
\hline$x_{8}$ & $8.4799 e-04$ & $7.4813 e-04$ & $x_{64}$ & $1.4134 e-05$ & $9.9173 e-06$ \\
\hline$x_{9}$ & $9.7387 e-04$ & $4.7122 e-04$ & $x_{72}$ & $1.6120 e-05$ & $5.3045 e-06$ \\
\hline$x_{10}$ & $1.1015 e-03$ & $1.5269 e-04$ & $x_{80}$ & $1.8131 e-05$ & $2.1047 e-08$ \\
\hline$x_{11}$ & $1.2305 e-03$ & $2.1017 e-04$ & $x_{88}$ & $2.0162 e-05$ & $5.9769 e-06$ \\
\hline$x_{12}$ & $1.3606 e-03$ & $6.2023 e-04$ & $x_{96}$ & $2.2208 e-05$ & $1.2736 e-05$ \\
\hline \hline
\end{tabular}



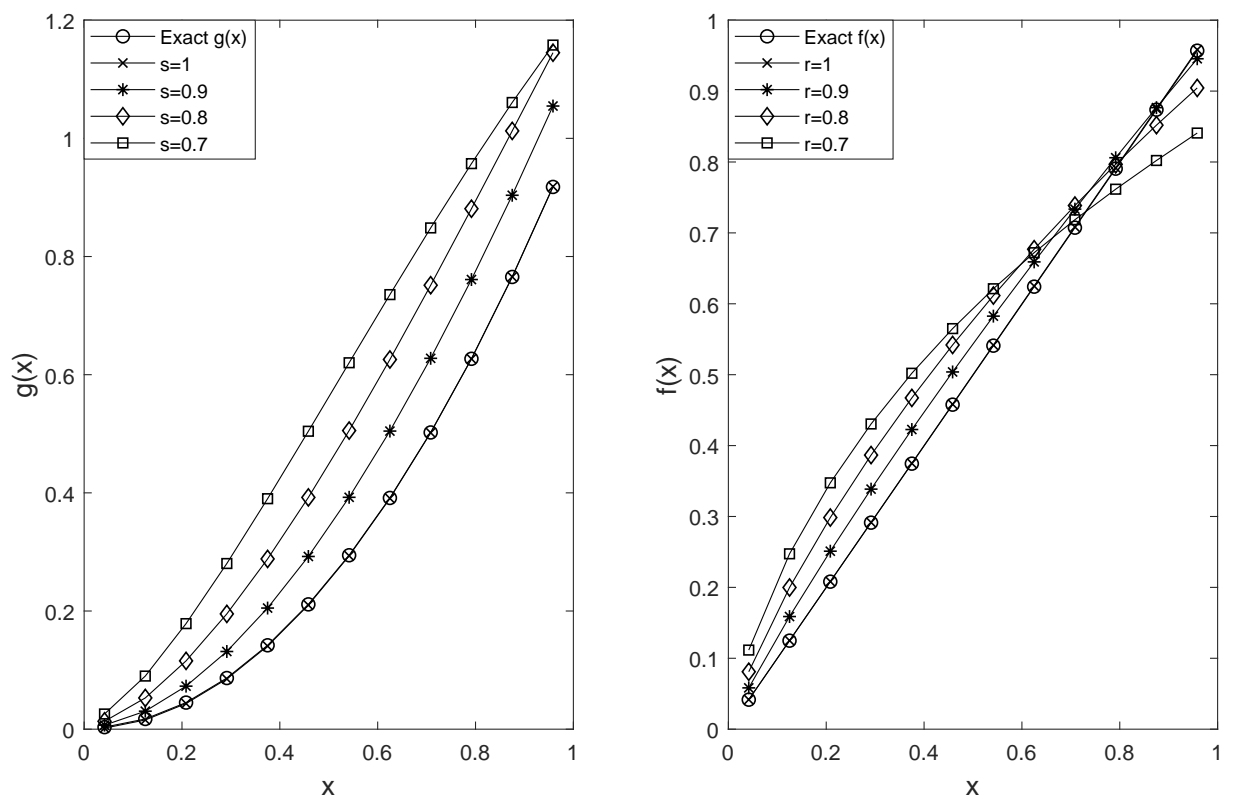

FiguRE 6. Numerical solution for different values of $r$ and $s$, when $k=2, M=6$ and $n^{\prime}=12$.
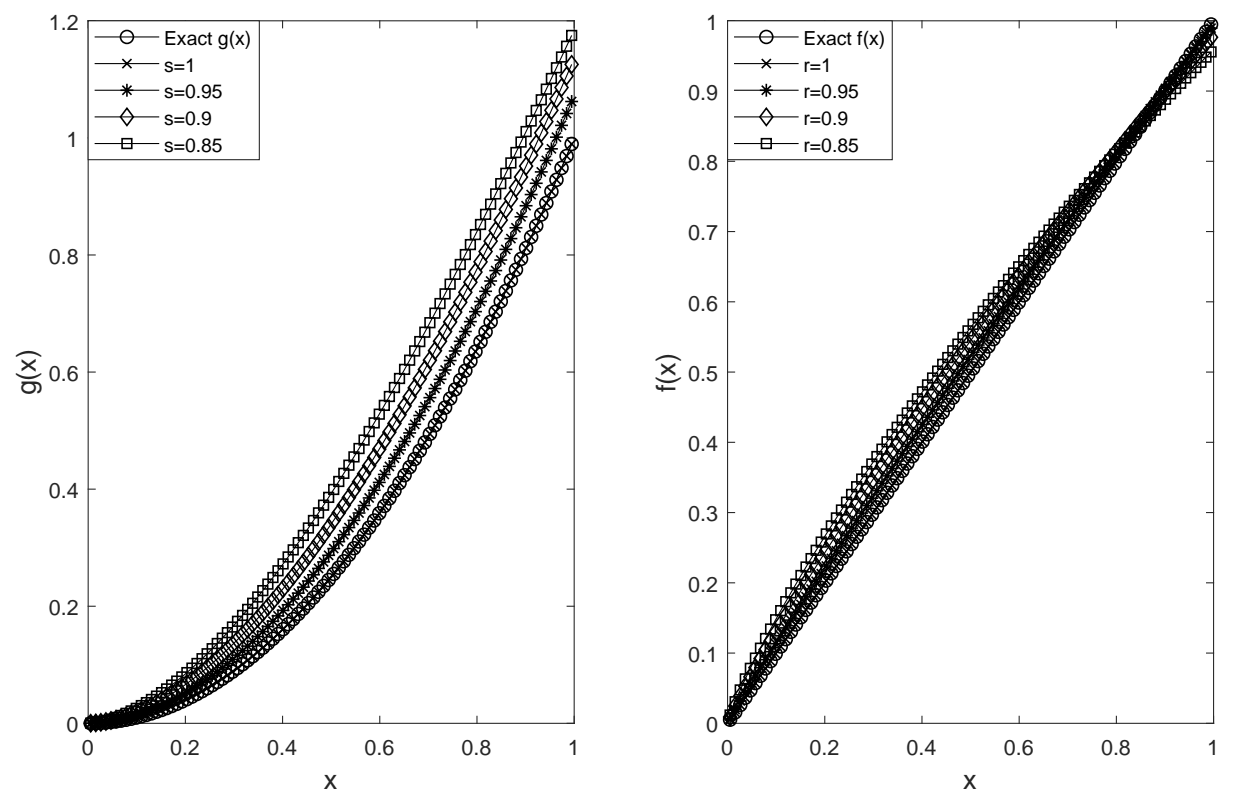

Figure 7. Numerical solution for different values of $r$ and $s$ when $k=6, M=3$ and $n^{\prime}=96$. 

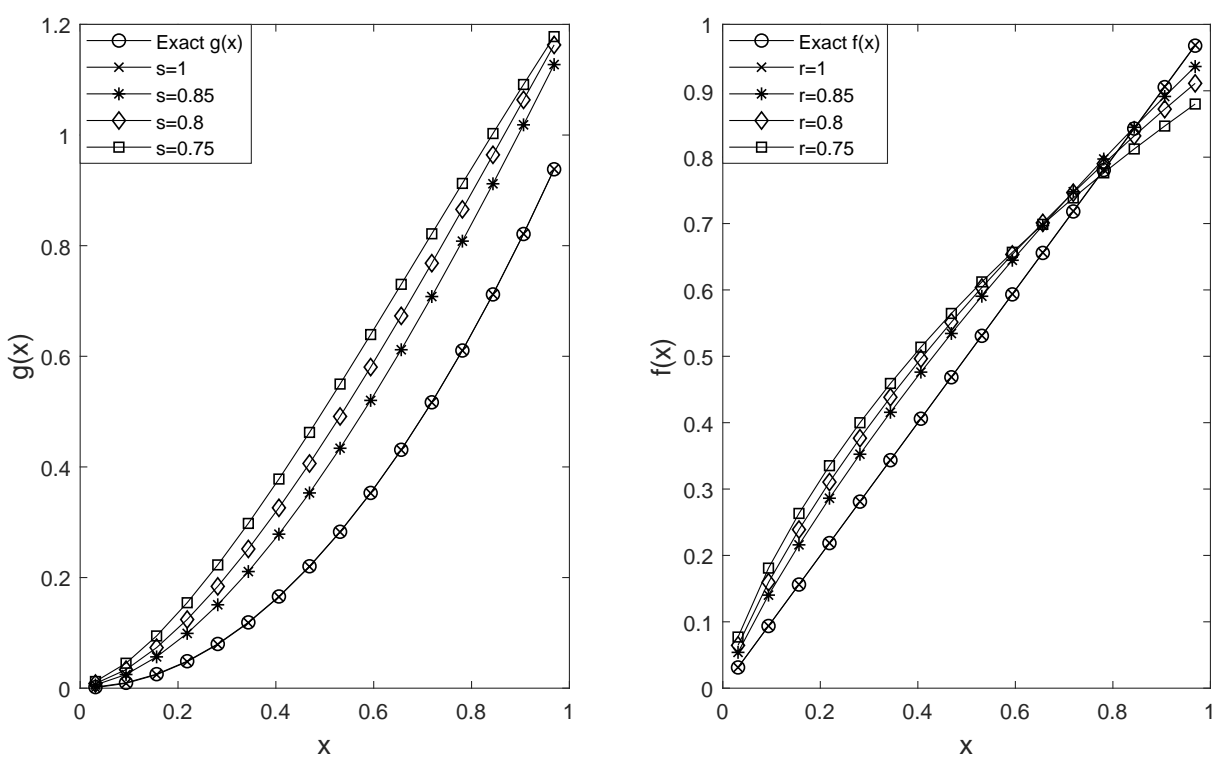

Figure 8. Numerical solution for different values of $r$ and $s$ when $k=3, M=4$ and $n^{\prime}=16$.
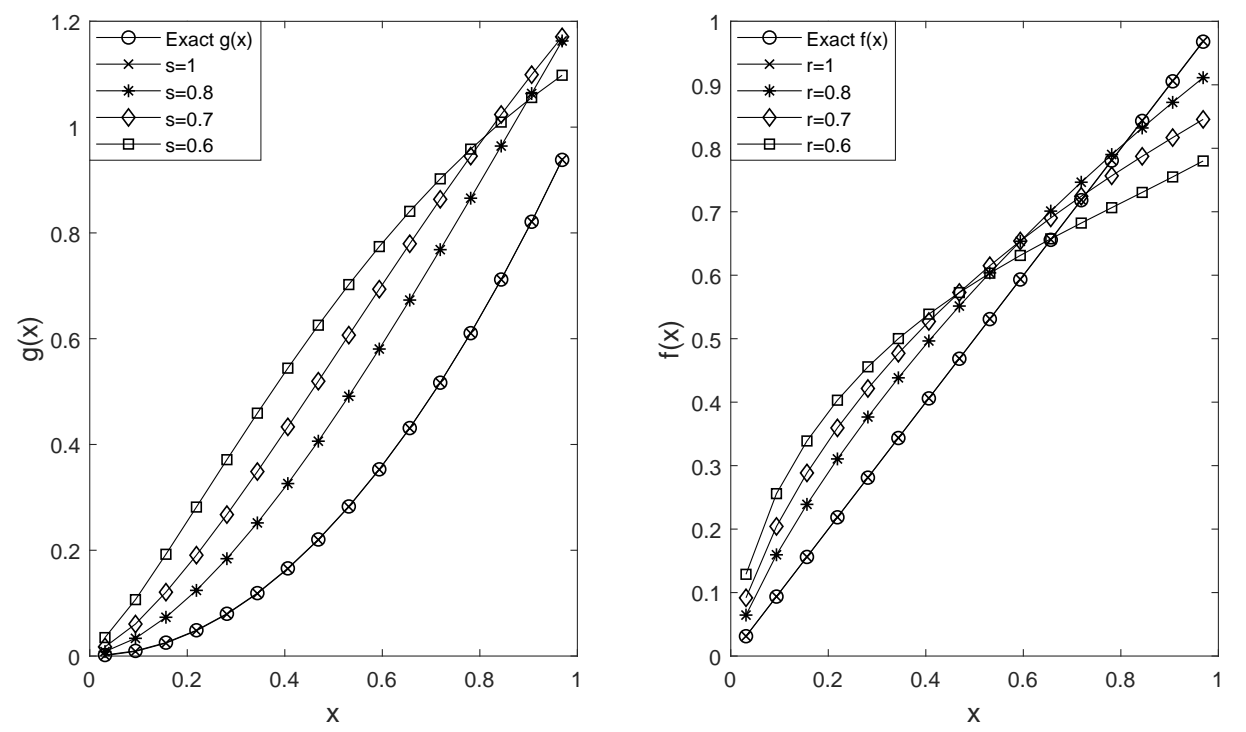

Figure 9. Numerical solution for different values of $r$ and $s$ when $k=3, M=4$ and $n^{\prime}=16$. 

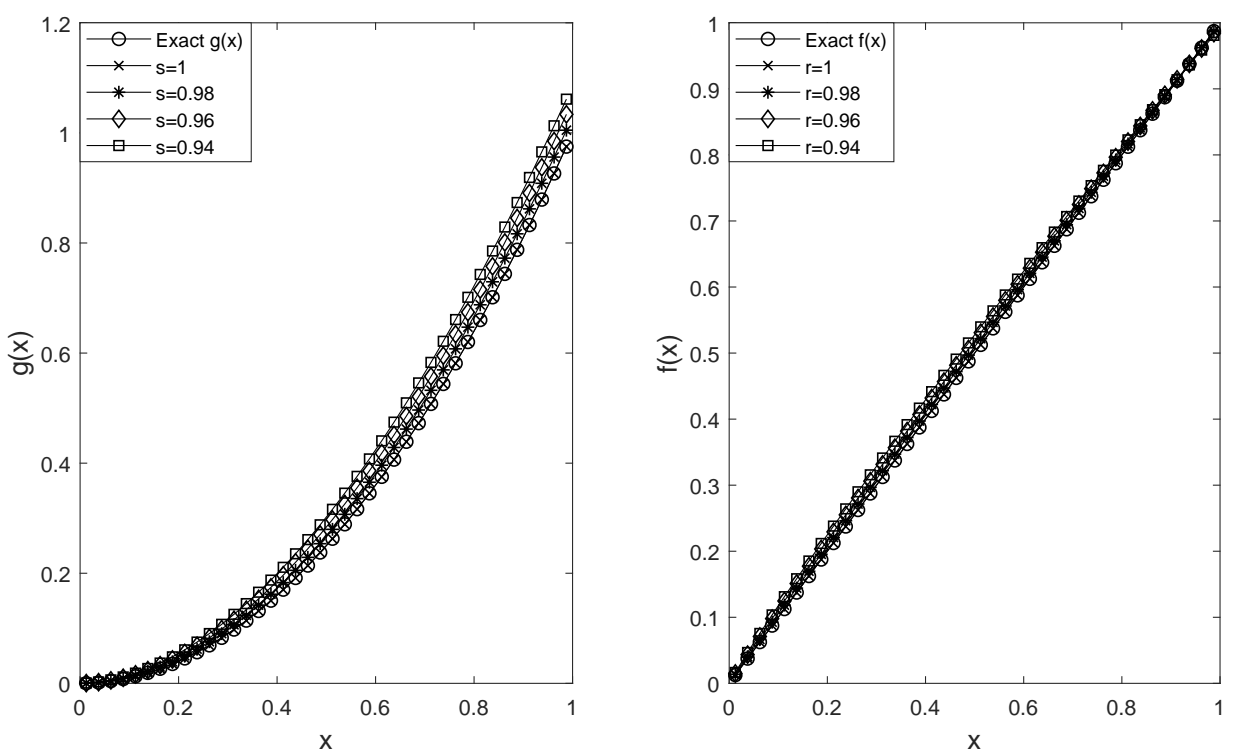

FiguRE 10. Numerical solution for different values of $r$ and $s$ when $k=4, M=5$ and $n^{\prime}=40$.

\section{Conclusion}

The main purpose of the presented article is introducing Legendre wavelets method for resolving coupled systems of FIDEs. As you saw, the numerical results obtained here, confirm its high accuracy degree.

The most noticeable profit of the mentioned method is converting complicated equations to simple ones, like we performed on examples. One of the best benefits of this procedure is having high exactness that you may have been recognized it according to the tables and figures.

\section{REFERENCES}

[1] A. Avudainayagam and C. Vant, Wavelet-Galerkin method for integro-differential equations, Appl. Numer. Math. 32(3) (2000), 247-254.

[2] E. Babolian and F. Fattahzadeh, Numerical solution of differential equations by using Chebyshev wavelet operetional matrix of integration, Appl. Math. Comput. 188(1) (2007), 417-426.

[3] C. K. Chur, Wavelets: A Mathematical Tool for Signal Analysis, Siam, Philadelphia, 1997.

[4] K. Goyal and M. Mehra, An adaptive meshfree spectral gragh wavelet method for partial differential equations, Appl. Numer. Math. 113 (2017), 168-185.

[5] M. H. Heydari, M. R. Hooshmanddasl and F. Mohammadib, Legendre wavelet method for solving fractional patial differential equations with Dirichlet boundary conditions, Appl. Math. Comput. 234 (2014), 267-276.

[6] M. H. Heydari, M. R. Hooshmanddasl, F. M. Maalekahaini and F. Mohummdi, Wavelet collocation method for solving multi order fractional differential equations, J. Appl. Math. (2012), Article ID 542401, 19 pages. 
[7] M. H. Heydari, M. R. Hooshmanddasl, C. Cattani and M. Li, Legendre wavelets method for solving fractional population growth model in a closed system, Math. Probl. Eng. (2013), Article ID 161030,8 pages.

[8] R. Hilfer, Applications of Fractional Calculus in Physics, World Scientific, Singapore, 2000.

[9] C. H. Hsiao and S. P. Wu, Numerical solution of time-varying functional differential equations via Haar wavelets, Appl. Math. Comput. 188(1) (2007), 1049-1058.

[10] H. Jafari, S. A. Yousefi, M. A. Firoozjaee, S. Momani and C. M. Khalique, Application of Legendre wavelets for solving fractional differential equations, J. Comput. Appl. Math. 62 (2011), 1038-1045.

[11] E. Keshavarz, Y. Ordokhani and M. Razzaghi, Bernoulli wavelet operetional matrix of fractional order integration and its applications in solving the fractional order differential equations, Appl. Math. Model. 38(24) (2014), 6038-6051.

[12] E. Keshavarz, Y. Ordokhania and M. Razzaghib, The Taylor wavelet method for solving the initial and boundary value problems of Bratu-type equations, Appl. Numer. Math. 128 (2018), 205-216.

[13] V. V. Kulish and J. L. Lage, Application of fractional calculus to fluid mechanics, Journal of Fluids Engineering 3 (2002), 803-806.

[14] S. Lal and V. K. Sharma, On wavelet approximation of a function by Legendre wavelet methods, Funct. Anal. Approx. Comput. 9(2) (2017), 11-19.

[15] Y. Li and K. Shah, Numerical solution of coupled systems of fractional order partial differential equations, Adv. Math. Phys. (2017), 14 pages.

[16] Y. Li and N. Sun, Numerical solution of fractional differential equations using the generalized block puls operational matrix, J. Comput. Appl. Math. 62 (2011), 1046-1054.

[17] F. Mohamad and C. Cattani, A generelized fractional order Legendre wavelet Tau method for solving fractional differential equations, J. Comput. Appl. Math. 339 (2017), 306-316.

[18] S. Momani and R. Qaralleh, An efficient method for solving systems of fractional integrodifferential equations, Comput. Math. Appl. 52 (2006), 459-470.

[19] I. Podlubny, Fractional Differential Equations, Academic Press, San Diego, USA 1999.

[20] M. Razzaghi and S. Yousefi, Legendre wavelets operational matrix of integration, Internat. J. Systems Sci. 32 (2001), 495-502.

[21] J. Wang, T. Z. Xu, Y. Q. Wei and J. Q. Xie, Numerical simulation for coupled systems of nonlinear order integro-differential equations via wavelets method, Appl. Math. Comput $\mathbf{3 2 4}$ (2018), 36-50.

[22] M. X. Yi, L. F. Wang and J. Huang, Legendre wavelet method for the numerical solution of fractional integro-differential equations with weakly singular kernel, Appl. Math. Model. 40 (2016), 3422-3437.

${ }^{2}$ Department of Mathematics,

Karaj Branch, Islamic Azad University, KaraJ, Iran

Email address: ezati@kiau.ac.ir

${ }^{1}$ Department of Mathematics,

Hamedan Branch, Islamic Azad University, Hamedan, Iran

Email address: razih.kaveh@iauh.ac.ir

Email address: n.karamikabir@yahoo.com

Email address: Yaghoobi@iauh.ac.ir

*CORRESPONDING AUTHOR 